\title{
PROCEEDINGS OF THE SOCIETY OF BRITISH NEUROLOGICAL SURGEONS: 55th MEETING
}

The 55th meeting of the Society of British Neurological Surgeons was held in Dublin from May 16 to 18, 1957, as a joint meeting with the Irish Ophthalmological Society (36th meeting). The two Presidents, W. R. Henderson (Leeds) and Alan J. Mooney (Dublin), occupied the chair in rotation.

\section{Some Neuro-Ophthalmological Problems}

Alan J. MoONey (Dublin) discussed a number of problems common to the ophthalmologist and the neurologist and illustrated these with individual case histories. He illustrated first two cases in which errors in diagnosis had been made. In the first an unnecessary cranial operation had been undertaken in 1935 whereas today investigation by cerebral angiography would have avoided the necessity. In the second, delay in operating on an acoustic neurinoma had resulted in blindness from secondary optic atrophy, and he stressed the fact that the presence of papilloedema constituted an urgent reason for definitive diagnosis, if necessary, by exploratory operation.

He then discussed the problem of congenital lesions of the optic discs simulating papilloedema. Such lesions were not rare and were sometimes, but not always, associated with hypermetropia. The presence of colloid bodies at the disc margin, fibrosis on its surface, and the absence of both a florid congested appearance and darkening of the veins after they pass over the disc margin should arouse doubts about the condition being pathological. Two cases were cited in which a mistaken diagnosis of papilloedema had been made in such circumstances, later investigations for an intracranial lesion proving negative. It was suggested that where the diagnosis of papilloedema was in doubt, repeated observations over a period of time might provide the true answer.

The difficulties inherent in diagnosis in patients with hypertensive retinopathy were discussed. Two cases were cited of this condition in one of which the diagnosis was true hypertension and in the other hypertension was complicated by an associated intracranial tumour, later removed at operation and found to be a metastatic carcinoma. It was suggested that where a patient was suffering from headaches without neurological signs and blurred discs, there was generally no urgency and repeated fundus observation would in many cases avoid the necessity for ventriculography or even craniotomy.

The presence of papilloedema in association with tuberculous meningitis was thought to be an indication for surgical treatment by third ventriculostomy and removal of adhesions around the optic nerves. In two of such cases treatment was successful, though in one vision was lost from secondary optic atrophy. Sometimes a serous papilloedema occurred, possibly associated with external hydrocephalus and in such cases conservative treatment was justified.
The occurrence of bilateral optic neuritis was mentioned and the difficulties in differentiating this condition from intracranial lesions stressed. Usually routine investigations, including skull radiographs and angiography, would provide an answer. It was also noted that refractive errors might confuse the diagnosis in some cases and lead to a misinterpretation of results in others. An example was given of visual failure in an acromegalic patient due to the onset of presbyopia and not to any involvement of the visual pathways.

Examples were given of pituitary tumours simulating retinal lesions and Adie's syndrome. It was stressed in connexion with pituitary tumours that pallor of the discs with bitemporal field loss was an indication for operation, possibly followed by $x$-ray therapy, whilst bitemporal field loss with normal discs indicated a retrochiasmal tumour. In such cases the operation risks were high and $x$-ray therapy was to be preferred.

It was noted in connexion with pituitary tumours that narrowing of the proximal part of the anterior cerebral artery was often associated with pre-chiasmal extension of the tumour. This might have some influence on the development of disc pallor and possibly of certain perimetric findings, the anterior cerebral artery being compressed between the optic nerves and chiasma below and the base of the frontal lobe above. It had been noted that the anterior cerebral artery recovered its normal calibre after removal of the tumour and this might have some connexion with field recovery and improvement in disc colour after operation.

The difficulties in distinguishing chronic glaucoma and toxic amblyopia from intracranial lesions were noted and illustrative case histories given.

The question of optic atrophy in tuberculous meningitis was then considered. Three case histories were cited. In one case, vision was recovered after operation with third ventriculostomy and removal of adhesions around the optic nerves, in another a similar operative procedure was apparently ineffective but vision began to recover four months later. In the third case, apparently similar to the others, vision improved markedly with simple anti-tuberculosis treatment. It was thought that compression of pial vessels by adhesions determined the loss of visual function and re-establishment of blood supply following timely removal of adhesions or the arrest of their development by modern therapy might account for the visual recovery. No connexion had been traced between the nature or amount of therapy and visual failure in these cases. 
Visual Field Changes Produced by $x$-Ray Treatment of Pituitary Tumours

M. Chamlin (New York) described the method of assessment and results of visual field studies in patients with pituitary tumours treated by $x$-ray therapy. From a group of 180 such patients, 40 were selected for study who could be followed adequately by visual field studies during treatment or immediately afterwards and for a reasonable number of months or years. The remaining 140 cases were discarded because they had been treated by surgery first and immediately afterwards by $x$-ray therapy, because adequate visual field studies could not be obtained, because they were seen on only one or two occasions, or because, although there was clinical evidence of a pituitary tumour, the visual fields were unaffected.

Most cases in the group of $\mathbf{4 0}$ were given a tumour dose of 3,000 r divided into three courses of $1,000 \mathrm{r}$ each. Each course was spread over four to six weeks with an interval of three to four weeks between, the total treatment covering some six months. During the course of treatment, patients were seen frequently and three functions assessed: visual acuity (Snellen), central visual field to $1 / 2000$, and peripheral field to $2 / 330$. If any of these functions deteriorated then treatment was diminished in dose or frequency until this function had improved. If these functions were maintained or improved then treatment was continued as outlined.

The results of treatment were evaluated in terms of 240 units of function, i.e., central visual acuity, central and peripheral fields in 80 eyes (40 patients). On this basis, function was improved in $50.8 \%$, maintained in $41.2 \%$, and deteriorated in $7.9 \%$. It was noted, however, that in 44 units of function this was normal and not capable of improvement. If these were subtracted from the total, then function was improved in $62.2 \%$, maintained in $28 \%$, and deteriorated in $9.6 \%$. In four cases surgical treatment was considered necessary later, two had large chromophobe tumours, and two cystic chromophobe tumours.

Improvement was evaluated in terms of episodes of improvement in the 240 units of function, 63 such episodes were noted. Of these, $44 \%$ occurred during the first year, $37 \%$ in the second year, $14 \%$ in the third year, and $5 \%$ in the fourth year. Of those occurring in the first year, only about one-half took place during or immediately after treatment; the remainder occurred at an average of four and a half months after treatment.

It was concluded that in assessing the value of $x$-ray treatment in pituitary tumours, provided no deterioration had taken place, one should allow at least four to six months after the completion of treatment before judging whether or not improvement will take place and at least two to three years before assessing the total amount of improvement.

\section{The Diagnostic Value of Orbital Angiography}

H. KRAYenbuhl (Zürich) discussed the clinical value of orbital angiography for the demonstration and differential diagnosis of orbital neoplasms and vascular abnormalities. The case material consisted of 117 cases 6 of unilateral or bilateral exophthalmos. In a series of 200 lateral carotid angiograms performed with the percutaneous technique of injection of $35 \%$ " diodrast," it was shown that only in $24 \%$ of the cases the ophthalmic artery and its branches were demonstrated. But by the use of a $60 \%$ contrast medium (Urografin-Schering) the ophthalmic artery was shown in $98 \%$ of the cases and in $74 \%$ the choroidal plexus of the eye was demonstrated. The latter was best seen two to five seconds after the beginning of the injection into the carotid artery in the neck which confirms the observations already made by Schurr (1951) and Decker (1955). As pointed out by Schurr, recognition of the orbital arteries and of the choroidal plexus is not easy; it is especially difficult to distinguish them from the outlines of the bone which overlies them. In his experience the stereoscopic views of the angiograms were of great help in the demonstration of these vessels. A few normal lateral carotid angiograms were shown to demonstrate the course of the ophthalmic artery and its branches and the appearance of the choroidal plexus.

As a second method of orbital angiography, he had developed, with his co-worker, G. Yasargil, orbital phlebography by puncture of the angular vein and injection with contrast medium. The percutaneous technique was described and the normal phlebogram shown.

Illustrative records were given of nine cases in which carotid angiography and angular venography helped in the diagnosis of orbital lesions. These included a melanoma, two retinoblastomata, one optic nerve glioma, one orbital neurinoma, one carotid-cavernous fistula, and one orbital varix. In two further cases the diagnosis was not verified by operation.

From the selection of this relatively small series it was noted that the results could not be used for generalization of the diagnostic value of orbital angiography, but, as Schurr (1951) had already pointed out, this method deserved the attention of ophthalmologists, radiologists, and neurosurgeons. With further experiences the method might be of use in the demonstration and differentiation of the nature either of orbital and periorbital neoplasms or of vascular abnormalities. However, from this series it seemed that the value of orbital angiography lay most likely in the demonstration of the pathological vascularization of the space-occupying lesion and not of the displacement of the ophthalmic artery and its branches.

\section{REFERENCES}

Decker, K. (1955). Fortsch. Röntgenstrahl., 82 (5), 667. Schurr P. H. (1951). Brit. J. Ophthal., 35, 473.

\section{Acute Visual Failure as a Neurosurgical Emergency}

A. UiHLEIN (Rochester, U.S.A.) discussed the neurosurgical aspects of acute visual failure. The common conditions leading to sudden visual failure were cited and included tumours in the pituitary, baso-frontal and parasellar region and of the visual pathways, carotid artery thrombosis and supraclinoid aneurysms, hydrocephalus, pseudotumour cerebri, chiasmal arachnoiditis, and injury. Three illustrative case histories were given.

Case 1.-A 64-year-old patient had harboured an 
unsuspected pituitary tumour for some time and symptoms, including visual failure, only began eight days before treatment. The left eye was blind and only spotty vision present on the right. Skull radiographs and angiography confirmed a diagnosis of pituitary tumour which was removed at operation. Vision in the right eye returned to normal and that in the left was greatly improved.

Case 2.-A 12-year-old boy was referred with a twoweek history of rapid visual failure in the left eye. The left eye became blind within two days of admission and vision in the right became blurred. Operative exploration of the optic nerves disclosed an haemangioendothelioma of the left optic nerve and left half of the chiasma with a very rare finding of intramedullary rupture of the left optic nerve. The left nerve and left half of the chiasma were resected. $X$-ray therapy was given later. There was no further loss of right vision.

Case 3.-A 10-year-old boy was referred with a history of head injury five weeks previously followed by headaches and gradual loss of right vision. Examination showed the right eye to be almost blind and a temporal hemianopia developed in the left later. Ventriculography showed a filling defect in the chiasmatic cistern. Operation disclosed an astrocytoma of the right optic nerve and right half of the chiasma which was removed. After this the left visual field improved and acuity returned to normal.

It was stressed that acute visual failure was more often than not due to an ophthalmological disorder. However, a neurological lesion of, or in the vicinity of, the optic pathways may be found and it was necessary to recognize the true cause as early as possible in order to prevent irreparable visual loss.

A thorough clinical investigation, including careful plotting of the visual fields and skull radiographs, was essential. Occasionally an apparently hopeless situation could be rectified, a possibility which justified the search for an operable lesion.

\section{Microdissection of the Capilla : Relationships in the Human Optic Nerve, Cnip 1 , and Tract}

C. A. ERSKINE (Dublin) desc i the microdissection of fixed tissues combined wit.. a new phase-contrast colour-contrast condenser. This was the first time the method had been used and was employed in this instance to investigate the capillary-glia relationship in the normal human optic nerve, chiasma, and optic tract. The operations were photographed in colour.

The questions invesitgated concerned the presence of a glial membrane in the optic nerve, chiasma, and tract, and if present its character and structure. In addition, an attempt was made to determine the relationship of the glial cells and their processes to the capillary wall and to the arteriole and venous adventitia; whether or not the glia differed in its degree of attac to these different vessels in the tissue investigated, whether it was possible to demonstrate a potential space between the vessel wall and glial structures (the so-called space of His), and whether or not a Virchow-Robin space passed as far as the capillaries in the nerve, chiasma, or tract.
It was concluded that glial elements in the form of a mesh separated the nerve fibres from the capillary at every point examined but that the presence of a glial membrane was uncertain. The presence of connective tissue around the endothelium seemed to determine the degree of fixation of the glial mesh, for the glia was attached to the adventitia whereas in the absence of connective tissue the glia was not fixed to the capillary endothelium. Thus a space of His could be demonstrated at this level. A Virchow-Robin space could be demonstrated directly in the septal region of the optic nerve in relation to the capillaries whereas this space could not be demonstrated at the capillary level in the chiasma and tract where perivascular pial connective tissue was usually absent. The presence of the Virchow-Robin space depended, therefore, on the amount of relative connective tissue and not on the size of the vessel.

\section{The Vascularization of the Primary Optic Pathways}

J. Francois, A. Neetens, and J. M. Collette (Ghent) reported the results of their investigations into the vascularization of the primary optic pathways by histological and microangiographic techniques. They had shown that the longitudinal capillary network of the optic nerve was normally continued towards the retina to form there a peripapillary network which anastomosed with the capillary network proper of the retina. This peripapillary network might originate from the pial plexus, the Zinn-Haller circle or from the ciliary system and formed a cilioretinal vascularization. Affection of this network probably explained the atrophic rings which were often seen around the disc in certain degenerative or atrophic diseases of the optic nerve.

The capillary and arterial vascularization was denser in and around the disc than in the retina or optic nerve itself and this probably explained the frequency and intensity of papillary oedema. The richness of vessels in the optic disc as well as the independence of the central retinal artery during its course in the opuc nerve was of great significance in the pathogenesis of functional disturbances in glaucoma.

Arteriosclerosis might produce lesions not only in the optic nerve but in other parts of the visual pathways. Disturbance of function could be produced by compression of fibres from hardened vessels, obliteration of a nutritive vessel, or by obstruction of capillary networks. Interruption of nutritive arterioles would have less effect in situations where they were numerous, as in the chiasma, or where there were numerous anastomoses as in the peripheral network of the optic nerve. If, however, the interruption affected a vessel which was isolated or partially an end artery then acute disturbance of function might result. It was considered that besides papillitis or optic neuritis of inflammatory or infective origin there might exist also an optic neuritis of "vascular" origin. This might be due to spasm or partial or total thrombosis of the central artery of the optic nerve which might produce a condition comparable to embolism of the central retinal artery. This type of condition was sometimes seen during temporal arteritis and was characterized by oedema or atrophy of the optic disc, lowering of central vision and central field defects. The 
peripheral field might be preserved since the peripheral nutritive vessels of the optic nerve might be unaffected.

In the intracranial portion of the optic nerve the precapillary and capillary vascularization was not only more irregular but was less dense temporally than nasally so that the temporal portion was more vulnerable than the nasal. Certain nasal defects of visual field could be more clearly explained by inhibition or arrest of the precapillary or capillary circulation than by the effects of pressure from sclerotic carotid arteries. The latter, if they were not very rigid, were more easily deformed than the optic nerve itself.

It was not thought possible that chiasmal function could be impaired by occlusion of one or even more arteries for it was profusely supplied by 11 arteries and a whole network of anastomotic arterioles, including the prechiasmatic arteriolar arcade. The disturbances of function should be attributed to an internal capillary affection due either to degeneration or, more frequently, to external compression.

Absence of hemianopia after ligation of the anterior choroidal artery for involuntary movements had caused some surprise. This could, however, be explained by a double vascular supply to the optic tract from the anterior choroidal artery and posterior communicating artery by means of hair-pin recurving precapillaries. The fact that hemianopia did sometimes follow such a ligation was probably explained by anomalies in the circle of Willis. They had observed complete or partial absence of the posterior communicating artery.

The capillary network in the lateral geniculate body was very dense, as in the retina and disc and must, therefore, frequently show oedema. The clinical disturbances resulting were, however, largely unknown at the present time.

\section{The Blood Supply of the Optic Nerve and Chiasma and its Clinical Significance}

Brodie HúGHes (Birmingham) discussed the clinical significance of present knowledge of the blood supply of the optic nerves and chiasma. It was noted that in the optic nerve there was a clear segregation of blood supply between the central and peripheral areas of the nerve and that final supply was based on fibre bundles. These facts were reflected in clinical material in several ways. Arteriosclerotic vascular disease of the optic nerve tended to produce two types of field loss, irregular peripheral indentation of the field, corresponding to involvement of the peripheral blood supply, and central fibre bundle defects due to involvement of the central supply. A common feature of vascular lesions in the optic nerve and of indirect injury was clear-cut horizontal hemianopias, usually in the lower field, sometimes in the upper. Examples of such field loss in arteriosclerosis, in hypertension, after gastric haemorrhage, in angiomastosis of the nerve, and after trauma were demonstrated. It was noted that anatomical studies had not supplied any explanation of this phenomenon.

The significance of central scotomata in optic nerve compression was then discussed. It was stressed that involvement of the field periphery was a late phenomenon although the periphery of the nerve was the area most subject to pressure; examples of early central and peripheral field loss were shown. It was concluded that involvement of the vascular supply of the nerve could not serve as an explanation of these facts and that stretching or kinking of the nerve fibres themselves must be the cause of such field disturbances.

The blood supply of the chiasma was briefly described and the central chiasmal artery to the antero-inferior region and the lateral chiasmal artery to the inferolateral area specially mentioned. Isolated involvement of the central chiasmal artery might produce upper bitemporal depression in the fields, and examples of such field loss in arteriosclerotic cases were shown. It was considered that damage to this artery might also explain some case of indirect chiasmal injury.

The significance of late preservation of the upper nasal fields in chiasmal compression was discussed and the various reported explanations mentioned. It was concluded that the preservation of the lateral chiasmal artery, immune to compression from a centrally placed tumour, was probably responsible for this feature of chiasmal compression. The rôle played by the anterior cerebral artery grooving the upper surface of the chiasma or optic nerves in association with tumour compression was discussed and illustrative operation sketches and field studies shown. It was concluded that while such a feature might account for nasal field loss in the early stages of compression, it did not provide a satisfactory explanation of the orderly sequence of field loss usually seen in chiasmal compression from tumour.

The phenomena of nerve fibre bundle defects and of preserved islands of temporal field in chiasmal compression were briefly referred to.

\section{Optic-disc Oedema in the Absence of Raised Intracranial Pressure}

M. J. Roper-Hall (Birmingham) described five cases referred to hospital as suffering from bilateral papilloedema. Investigations were essentially negative, at least as an explanation of the disc-head oedema. In none of them was there any loss of visual function, such as might be associated with orre neuritis, and the intracranial pressure was provesi va be within normal limits by lumbar puncture after prelink ..... in investigations had determined that such was a safe piocedure.

One was a case of arterial hypertension, two had incomplete central retinal vein obstruction, one case was an example of papilloedema associated with benign intracranial hypertension in a regressing phase, and the last case showed relative hypertension of cerebral circulation associated with a posterior communicating artery aneurysm. They formed part of a group of 600 cases admitted to hospital for intracranial conditions, 11 of which were classified as benign intracranial hypertension.

A plea was made for the strict definition of the term papilloedema. Tintirature revealed that oedema of the discs in blood dyscrasias and hypoparathyroidism was probably true papilloedema since whenever recorded the cerebrospinal fluid pressure was raised. Normal or low cerebrospinal pressures were recorded with disc oedema in malignant hypertension in the Guillain-Barré syn- 
drome and in traumatic hypotension. Simulated bilateral papilloedema with drusen of the optic nerve was mentioned.

There was experimental and clinical evidence that with a rise of intracranial pressure there was a compensatory rise of pressure in the arteries and veins at the disc-head. This could be fully compensating and prevent the onset of papilloedema. As papilloedema appeared the arterial diastolic pressure was found to fall.

It appeared that optic disc oedema could occur without a raised cerebrospinal fluid pressure. Compression of the central retinal vein in the subarachnoid space could be ruled out as a factor in the production of oedema in these cases.

After considering some other experimental facts it was suggested that in these cases gradual reduction of nutrition and anoxia of the nerve might be sufficient to lead to oedema without loss of function.

The suggestion was put forward that, by a simple alteration of the arterial and venous pressure ratios at the optic disc, papilloedema could be accurately simulated thereby lending support to the theories which suggested similar conditions in the aetiology of true papilloedema.

\section{Ocular Aspects of Endocrine Exophthalmos}

G. I. Scotr (Edinburgh) discussed the ocular aspects of endocrine exophthalmos from the point of view of the clinician. The ocular signs of acute thyrotoxicosis were contrasted with those of malignant or oedematous exophthalmos and special reference was made to two uncommon, but important, aspects of the latter condition.

In the first place, there was the type of case in which the exophthalmos might remain unilateral over a long period and in which the diagnostic difficulty lay in deciding whether one was dealing with an orbital tumour or a case of endocrine exophthalmos. In the second place, there was the occurrence of visual field defects in cases of malignant exophthalmos, likewise raising a suspicion of orbital tumour.

The case notes of two patients were presented, in the first of whom the ocular signs remained unilateral for over a year, and in the second of whom only one eye was affected, the other eye remaining unaffected over a period of five years.

Two cases of involvement of the fields of vision in malignant exophthalmos were described. In the first, there was a bilateral central scotoma which extended with increasing involvement of macular vision to such an extent that decompression of the orbits was considered advisable, after which there was a dramatic restoration of vision in both eyes. The second case also showed a dense central scotoma in each field, the effect upon visual acuity being less severe than in the first case and spontaneous recovery taking place at the end of a year from the start of symptoms.

The two cases in which the ocular signs were unilateral were treated by irradiation of the retroocular tissues, the patients receiving 3,000 rads in three weeks and the field being restricted so as to exclude, as far as possible, the pituitary fossa.
It was suggested that irradiation of the orbit was a valuable method of treatment in cases with severe and persistent proptosis. It was emphasized, however, that malignant or oedematous exophthalmos appeared to be a self-limiting disease and that active measures such as irradiation of the orbit or decompression were only indicated if the exophthalmos became manifestly "malignant" from the point of view of visual function.

\section{Invasion of the Optic Chiasma and Optic Nerves by Neighbouring Gliomas}

Sir Geoffrey Jefferson, E. Pollak, and Peter O. YATES (Manchester) discussed some pathological problems of gliomas invading the optic nerves and chiasma. Two aspects of the problem were considered, first the method by which these tumours reached the optic nerves and chiasma, and secondly, the nature of the tumours.

The type of tumour encountered was determined to a large extent by the ease of access of any particular variety to the chiasma. The oligodendrogliomas, for instance, which commonly grew in the cerebral hemispheres, were not likely to be in a position to invade the chiasma. On the other hand, gliomas of the optic nerve might easily invade the chiasma or even the opposite optic nerve. Tumours in the frontal lobes would necessarily have to pass through the lamina terminalis or basal ganglia whilst temporal lobe gliomas might grow directly into the optic tract and thence to the chiasma. Tumours from the thalamic regions and those arising from the hypothalamus and infundibulum would have to grow downwards through the floor of the third ventricle.

Quite a different mode of invasion was the indirect route via the meninges. In such instances the tumour might arise anywhere within the brain and in some cases it had been impossible to determine the primary site of growth. They had sometimes encountered a condition of diffuse invasion of the arachnoid by glial tumour bearing a superficial resemblance to purulent meningitis. In such meningeal gliomatosis there was a tendency for tumour growth to concentrate in the basal cisterns and around the chiasma. The tumours which spread in the subarachnoid space were usually histologically malignant with the exception of the optic nerve glioma and tumours of a similar type growing elsewhere. These appeared to grow normally in the subarachnoid spaces and were usually closely mixed with a considerable amount of collagenous fibrous tissue.

The tumours most likely to invade the chiasma directly from above were those arising in closely related structures, particularly the hypothalamus and floor and walls of the third ventricle. Occasionally, these were welldifferentiated astrocytomas similar to those commonly found in the cerebral hemispheres of adults. More commonly they were slowly growing tumours and often started in childhood. Histologically they were similar to the infundibulomas described by Globus (1942). He had been impressed by the way in which dense glia surrounded blood vessels and resembled the portal vascular system of the infundibular region. Such perivascular sheaths of glia were not, however, confined to tumours of the infundibular region but were encountered also in the optic nerve gliomas and in the cerebellar astrocytomas 
of childhood. They had also been referred to as piloid astrocytomas by Elvidge, Penfield, and Cone (1935) and Russell (1950) but had many features which were not found in the ordinary astrocytomas of the cerebral hemispheres. They were the only gliomas more common in females and the cells found in them had a very strong tendency to form bipolar fibrous extensions, often grouped together in hair-like tresses. Rosenthal's fibres, which were an elongated, rounded and strongly acidophilic form of glial fibre, were common in this group and had also been encountered in the subependymal region in relation to a group of Rathke pouch tumours studied with Grcevic. Since the cytological origin of this group of tumours was in doubt the simple descriptive name of piloid glioma was to be preferred.

\section{REFERENCES}

Elvidge, A., Penfield, W., and Cone, W. (1935). Res. Publ. Ass. nerv. ment. Dis., 16, 107 .

Globus, J. H. (1942). J. Neuropath., 1, 59.

Russell, D. S. (1950). Postgrad. med. J., 26, 109.

\section{Visual Field Changes following Anterior Temporal Lobectomy}

M. A. FALCONER and J. L. WiLSON (London) reviewed the visual field changes following anterior temporal lobectomy in 50 patients suffering from intractable temporal lobe epilepsy. The extent of the operations ranged from 4.5 to $9 \mathrm{~cm}$. as measured from the temporal pole along the infero-lateral border of the lobe. In all cases the tip of the temporal horn of the ventricle was opened into at operation.

Subsequently in each case there was a visual field defect which in many instances was too slight to be detected by confrontation testing, but which could be elicited by detailed quantitative perimetry. The visual field defect was always congruous, affecting the contralateral homonymous fields. The extent of the visual field loss, however, varied considerably from case to case and had no hard and fast relationship to the extent of temporal lobe removal as measured along the inferior border of the lobe.

In the slightest degrees of hemianopia the loss was apparent only in the upper part of the contralateral homonymous fields immediately adjacent to the vertical meridian. Then with increasing severity of hemianopia the loss spread in a sector-shaped fashion towards the horizontal meridian. In even more severe hemianopia the loss extended downwards below the horizontal meridian, again in sector-shaped fashion towards the lower part of the vertical meridian. Macular vision tended to be spared except sometimes when the hemianopia was greater than a quadrant.

There were no significant differences in the range of extent of homonymous hemianopia after excisions of $6 \mathrm{~cm}$. or less and after excisions of between 6.5 and $7.5 \mathrm{~cm}$. The chances of there being an upper quadrantic hemianopia or less was in the order of 6 to 1 . Such a degree of hemianopia usually did not inconvenience patients. With excisions of $8 \mathrm{~cm}$. or more, however, the chances of a hemianopia greater than a quadrant increased and such a degree of hemianopia constituted an appreciable disability.
These findings ran counter to the prevailing view expressed in the literature (largely on observations made on tumour material) that lesions of the anterior temporal region produced incongruous homonymous field defects. Instead they lent support to the views of Meyer (1907) that the lowermost fibres of the optic (geniculo-calcarine) radiation loop round the tip of the temporal horn and then pass backwards inferior to the ventricle to reach the calcarine cortex. They also reinforced the more detailed views of Spalding (1952) on the anatomy of the optic radiations based on the effects of gunshot wounds.

\section{REFERENCES}

Meyer, A. (1907). Trans. Ass. Amer. Phys., 22, 7.

Spalding, J. M. K. (1952). Journal of Neurology, Neurosurgery, and Psychiatry, 15, 99, 169.

\section{Pseudo-tumours of the Orbit}

HARVEY JACKSON (London) discussed the clinical, pathological, and surgical features in a series of orbital pseudo-tumours. The general use of the term " pseudotumour" was discussed and it was stressed that in the orbit the term was used for a precise form of pathology, a granulomatous mass, probably of infective origin. Examples due to tuberculosis and syphilis might occur. A brief account was given of the history of the condition. The findings in a personal series of 36 cases were then given. These had formed $12.3 \%$ of a series of 293 cases of proptosis and it was the commonest single condition encountered in the series. The average age was 47.6 years, the youngest being 24 years. The condition was twice as common in the female and all of four bilateral cases were of this sex.

The pathological features were described as being those of a chronic granuloma with lymphocytes in follicular formation, plasma cells, and occasional eosinophil cells. Fibrous tissue was present in varying amounts. The ocular muscles were affected, the inferior rectus and inferior oblique most commonly, striations disappeared and the fibres stained poorly. The mass was often within the muscle cone but occasionally attached to the orbital periosteum, most commonly on the medial wall or floor. This finding suggested that air sinus infection might play a part in aetiology. No specific organisms were recovered in any case.

Proptosis was always present and might be of long duration; occasionally the history was a short one and differentiation from malignant disease necessary. Pain was also a common feature and necessitated differentiation from malignant tumours. Variability in signs was also very common but was not confined to this condition and had been observed in haemangiomas of the orbit. Exacerbation of signs often coincided with some general illness in the patient or with menstruation. External ocular movements were usually affected due to muscle infiltration by the granuloma; evidence of nerve paresis was not encountered. The ocular fundi were usually normal though gross papilloedema, retinal oedema, and optic atrophy had been seen. Diplopia was noted in half the cases but disturbances of visual acuity and visual fields were exceptional. Occasionally an orbital mass was palpable, usually on the medial side, but there were no 
features by which to distinguish such a mass from other orbital lesions.

Extensive clinical investigations were usually carried out but these produced no positive features and merely served to exclude other conditions. Radiological studies were not often helpful and merely showed blurring of detail in soft tissues. In two cases widening of the sphenoidal fissure was encountered due to extension of the granuloma through the fissure.

The diagnosis could often be made on clinical grounds alone; where there was doubt then biopsy of the mass was advisable. Medicinal treatment was the first choice and iodides, mercury, and arsenic were given in the form of Donovan's solution (mist. hydrarg. et arsen. iod.). In many cases this resulted in a resolution of the condition, impairment of upward ocular movement being the only common residual sign. When this treatment failed cortisone could be given a trial. Deep $x$-ray therapy had been advocated but no case in this series had been so treated. Occasionally tarsorraphy might be required to protect the cornea. Where medicinal therapy was ineffectual, resort to surgery might be necessary. The procedures adopted included orbital decompression and the local removal of masses.

Various points in the paper were illustrated by four detailed case histories.

\section{Epidermoids of the Orbit}

P. C. Carey (London) reviewed the clinical picture, diagnosis, and treatment of epidermoid and dermoid tumours of the orbit. This was based on a series of five epidermoid and two dermoid tumours encountered in a series of 293 cases of exophthalmos seen on the neurosurgical service of Mr. Harvey Jackson.

The tumours consisted of two parts, a capsule and its contents. The capsule was usually opaque, white and glistening or, in the case of dermoids, might vary from yellow to brown. The contents of the epidermoids consisted of concentric laminae of keratin and epithelial debris which had a soapy or waxen appearance. Dermoid tumours might contain in addition, sebaceous material, hair, and oily fluid.

Most investigators agreed that both epidermoid and dermoid tumours developed from misplaced epithelial rests. Others had suggested that the type of tumour arising was determined by the degree and embryonic antiquity of the rest. If only squamous epithelium were included then the tumour was an epidermoid; if, however, the rest contained epithelium and dermis then it might give rise to a dermoid tumour with fat cells, sebaceous glands, sweat glands, and hair follicles. Though the presence of hair had been regarded as pathognomic of dermoid tumours, cases had been recorded in which, although hair was present, the tumours were otherwise typical of epidermoids; these had been considered as transitional forms between the dermoid and epidermoid. It had been held that in the case of dermoid tumours, symptoms occurred earlier in life and were more likely to be associated with other congenital defects.

It was suggested that a common term to include the two tumours, such as pearly tumour or choleasteatoma, was inadvisable and that it was better to name the growth by its essential features, epidermoid or dermoid as the case might be. It was appreciated that owing to the technical difficulties in surgical excision these tumours might often have to be removed in a fragmentary manner so that only parts of the capsule might be submitted to histological examination.

Hormone Replacement Therapy in Craniopharyngiomata

A. S. KERR (Liverpool) reviewed the results of treatment in 27 cases of craniopharyngiomata seen during the past 10 years. Nineteen patients were known to be dead, two could not be traced, and only six were known to be still alive. The longest known survival was four and a half years.

All standard forms of treatment seemed to be equally ineffective from a curative point of view. Several patients had more than one type of procedure. Of nine in whom a cyst was tapped, all were dead with a longest survival of three and a half years; of six who had Torkildsen's operation only two were alive and one of these was not well 12 months later; of eight with partial removal, five were dead and one was unknown, whilst the two survivors each had further treatment, one with radiotherapy and one by complete removal. Of eight treated by radical removal, four were dead and the longest survival to date was four and a half years.

The factors which appeared to militate against successful surgery were (1) damage to the hypothalamus, and (2) damage to the maldeveloped anterior lobe of the pituitary gland. The former often manifested itself in hyperthermia in the immediate post-operative period and the introduction of hibernation (or hypothermic) techniques offered a measure of control of the patient's body temperature in the post-operative period.

Apart from clinical evidence, an assessment of diminished anterior pituitary function could be gained from laboratory tests, of which Thorn's original test seemed to have the greatest practical value. If adrenal cortical function is low, as judged by a poor response in the blood eosinophil count to an injection of A.C.T.H., it may be stimulated by repeated injections of A.C.T.H. for a week or two and the patient thereby strengthened to withstand the stress of the post-operative period. Alternatively, if the adrenal cortex cannot so be stimulated, the operation could be rendered safer by giving $100 \mathrm{mg}$. of cortisone pre-operatively. Most patients would probably require cortisone therapy in the postoperative period and this could be gradually reduced in amount during the first 10 to 14 days.

Some patients might need a maintenance dose of about $12.5 \mathrm{mg}$. of cortisone daily for an indefinite period. It was to be regretted that, as yet, there was no satisfactory preparation of growth hormones freely available.

Of the eight cases of total removal quoted above, five were operated upon under hypothermia and four of these had pre-operative hormonal assessment. Two died in a few days despite these precautions but there was a reasonable hope that the three survivors might be regarded as cured.

The removal of some extensive craniopharyngiomata would appear to be an impossible technical feat but palliative treatment was so ineffective that our aim should 
be a radical removal whenever possible. This was only justifiable, however, if the operation could be rendered safer than it was at present and the introduction of hypothermia and of hormonal control seemed to be steps in the right direction.

\section{Some Problems presented by Haematogenous Brain Abscess}

E. J. Newton (Birmingham) discussed the early development and experimental production of haematogenous brain abscesses.

Lesions in a case of bacterial endocarditis indicated that miliary cerebral abscesses resulted from rupture of capillaries which had been embolized by bacteria. Infarction did not appear to be a necessary event in the pathogenesis of these abscesses. There was evidence that brain abscesses arising from pulmonary suppuration might similarly develop from bacterial emboli arrested in cerebral capillaries.

Experiments undertaken in collaboration with Dr. Nancy Laughton were described. Attempts had been made to produce brain abscesses in rabbits by carotid injection of one or the other of two strains of nonhaemolytic streptococci, each isolated from a haematogenous brain abscess in a different human being.

An 11-day-old brain abscess occupying the thickness of the white matter in the parietal region had been produced. This showed early encapsulation and was very close to the lateral ventricle at one point. Many streptococci were demonstrable in the abscess by Gram's stain. Numerous small gliotic foci were also present in this brain bilaterally. They were commonest in the cortex but were also seen in the thalamus. It was assumed that all these lesions were embolic because the organism underwent spontaneous aggregation in broth culture and had been injected in this form.

The second streptococcus strain showed a similar tendency to spontaneous aggregation but the aggregates formed were smaller. Simple carotid injection of this organism into rabbits did not produce cerebral lesions. Embolic lesions were finally produced in one animal which had received an intravenous injection of serum containing specific agglutinins for the streptococcus immediately before the carotid injection. Eleven days later many gliotic foci were present in the cerebral cortex and one larger focus of active infection was found in the parietal white matter. This contained streptococci and polymorphonuclear leucocytes but otherwise had features of a granuloma rather than an abscess. Specific agglutinins to this streptococcus were present in the serum of the patient from whom it had been isolated and were effective in a serum dilution of 1 in 640 .

It was suggested that agglutinins might sometimes be an aetological factor in the pathogenesis of haematogenous brain abscess in man. 